\title{
One-day-ahead daily power forecasting of photovoltaic systems based on partial functional linear regression models
}

\author{
Guochang Wang ${ }^{a}$, Yan $\mathrm{Su}^{b}$, Lianjie $\mathrm{Shu}^{c}$ \\ ${ }^{a}$ College of Economics, Jinan University, GuangZhou 510632, China \\ ${ }^{b}$ Department of Electromechanical Engineering, University of Macau, Taipa, Macau \\ ${ }^{c}$ Faculty of Business Administration, University of Macau, Taipa, Macau \\ ( ${ }^{c}$ Corresponding author, E-mail: ljshu@umac.mo, Tel.:853-88224741, Fax:853-88222377)
}

\begin{abstract}
The intra-day time-varying pattern of solar data is more informative than the aggregated mean daily data. However, most of the traditional forecasting models often construct the 1-day ahead daily power forecast based on its historical daily averages but ignore the information from its intra-day dynamic pattern. Intuitively, the use of aggregated data could cause certain loss of information in forecasting, which in turn adversely affects forecasting accuracy. In order to make use of the valuable trajectory information of the power output within a day, this paper suggests a partial functional linear regression model (PFLRM) for forecasting the daily power output of PV systems. The PFLRM is a generalization of the traditional multiple linear regression model but enables to model nonlinearity structure. Compared to the neural network models that are often criticized by the requirements of past experience and reliable knowledge in the design of network architecture, the suggested method only involves a few parameter estimates. A regularized algorithm was used to estimate the PFLRM parameters. It is shown that the regularized PFLRM improves the forecast accuracy of power output over the traditional multiple linear regression and neural network models. The results were validated based on a $2.1 \mathrm{~kW}$ grid connected PV system. Keywords: Partial Functional Linear Regression, Solar Irradiance, Photovoltaic System, Efficiency
\end{abstract}




\section{Nomenclature}

$s$ or $t$

$y$

$x(s)$ or $x(t)$

$\mu(t)$

$\mathbf{Z}$

$\alpha$

$\beta(t)$

$\gamma$

T

$\varepsilon$

$\boldsymbol{\xi}_{i}$

K

$G(s, t)$

$\lambda_{k}$

$\phi_{k}(s)$

$c_{k}$

$\hat{\boldsymbol{\xi}}_{i}\left(\right.$ or $\left.\hat{\lambda}_{k}, \hat{\phi}_{k}(s), \hat{\beta}(t)\right)$

$\hat{G}(s, t)$

$\hat{y}_{i}$

$n$

$t_{i j}$

$U_{i j}$

$e_{i j}$

$x_{i}\left(t_{i j}\right)$

$L_{i}$

$\hat{\mu}(t)$

$\hat{c}_{k}$

$\hat{\gamma}$ time period

response

functional predictor

mean of $x(t)$

a vector of covariates

the intercept term for the PFLRM

regression coefficient function

regression coefficient vector

the time set

random error

functional principal component (FPC) score

number of leading components selected

covariance function

the $k$ th eigenvalue

the $k$ th eigenfunction

the $k$ th basis coefficient

estimate of $\xi_{i}$ (or $\left.\lambda_{k}, \phi_{k}(s), \beta(t)\right)$

estimate of $G(s, t)$

fitted value of $y_{i}$

sample size

the observation time of the $j$ th observation in day $i$

the recorded power output at time $t_{i j}$

measurement error

the $i$ th realization of $x(t)$

number of observations in day $i$

estimate of $\mu(t)$

estimate of $c_{k}$

estimate of $\gamma$ 


\section{Introduction}

In recent years, introduction of an alternative energy resource has been expected. Due to the advantages of being clean, abundant, and inexhaustible, solar energy has received gradually increasing attention as one of the best solutions for the alternative energy resources, and photovoltaic (PV) technology has been rapidly developed recently. PV systems become more and more popular in grid-connected applications rather than being established in remote areas [1]. However, the power output of a PV system is not constant but depends on solar irradiation and weather conditions. For a PV system, there are many factors that can influence the power output such as solar irradiance, temperature, insolation, and installation angle. Due to the variability of solar irradiance and environmental factors, the power output of a PV system is dynamically changing with time.

The variability of power output not only adversely affects the stability of the electrical system being connected but also may increase operating costs for the electricity system by increasing requirements of primary reserves [2]. For this reason, accurate prediction of power output is an important task to improve the integration stability of output of a solar PV system into electric grid and to help producers to implement operations strategy in an efficient way. The forecast of power output can be made at different time scales for different purposes. The short-term like intra-hour forecasts are often relevant for dispatching, regulatory and load following purpose [3]. However, the 1-day ahead forecast of the PV system is often required by the end-users such as energy traders for the purpose of trading the electricity market and transmission system operators for operational planning. This paper limits discussion to the accurate 1-day ahead forecast of PV power output.

A lot of research has been devoted to the development of forecasting tools for predicting PV power output with good accuracy. These forecasting tools generally fall into two categories, indirect and direct approaches. The indirect approach involves a two-step procedure for forecasting PV power output. In the first step, the solar irradiance is forecasted based on various approaches, including time series models, artificial neural networks, support vector machines, and others. For example, Lopez and Cardona [4] and Martín et al. 
[5] discussed the use of time series models for predicting global irradiance while Reikard [6] made a comprehensive comparison of time series models for predicting solar radiation at high resolution. Another frequently used method to forecast solar irradiance is based on artificial neural network. A sample of research in this respect includes [7]-[11]. Chen et al. [12] and Wu and Liu [13] employed support vector machine to estimate monthly solar radiation from measured temperatures. Cao and Lin [14] proposed a new model for forecasting global solar irradiance based on diagonal recurrent wavelet neural network. In the second step of the indirect approach, the forecasted solar irradiance and temperature data are often used as inputs in the commercial simulation softwares such as TRNSYSM [15], PVFORM [16] and HOMER [17].

The second category aims at directly predicting the power output based on some prior information or readily accessed data. Due to the similarity of forecasting solar irradiance and power output, it is straightforward to extend the methods for forecasting solar irradiance to the forecast of power output of PV systems. A list of research on the use of time series models for direct forecasting power output includes [3, 18, 19, 20] . A sample of research on the use of neural network models for this purpose can be referred to [21]-[25].

With rapid development and advancement in sensor and data acquisition technology, the solar data are often recorded at high frequency such on the basic of one minute, which can be also seen from the underlying data set collected from a $2.1 \mathrm{~kW}$ grid connected PV system. The high frequency solar data depicts the time-varying pattern (or trajectory) of the power output generated within a day. Clearly, the intra-day time-varying pattern of the power output is more informative than its daily average. However, most of the traditional methods construct the 1-day ahead forecast of power output based on the aggregated data, i.e., the historical daily power output, but ignore the valuable intra-day information. Intuitively, the intra-day trajectory of power output could be used to improve the forecast accuracy. Motivated by this, this paper suggests a new model based on the partial functional linear regression model (PFLRM) for generating the 1-day ahead forecast of the daily power output of PV systems. Although the PFLRM has been widely discussed in the statistical literature and successfully employed in other applications [27, 28], it has been less studied 
for forecasting power output.

The PFLRM is a generalization of both the classical multiple linear regression model and functional linear regression model, which has been proved to be a powerful tool in forecasting [26]. It can include function-valued random variable and some real-valued variables as predictor variables. Therefore, it is natural to apply the PFLRM to forecast the power output of PV systems by treating the intra-day trajectory of power output of the PV system in the previous day as a functional predictor and some real-valued climatic variables as covariates in the model. The PFLRM maintains simplicity as the traditional multiple linear regression model but provides more flexibility than the latter to model the nonlinear structure in the data. As compared to the completely data-driven forecasting models such as neural network models, the PFLRM achieves certain simplicity. The PFLRM often involves only a few parameter estimates that are entirely driven by the regression data. Instead, the design of neural network models often involves the design of network architecture and the selection of a good learning algorithm. There are many parameters that need to be prespecified. This heavily relies on past experience and is subject to trial and error processes since the optimal configuration is not known a priori. This is a limitation of neural network models often criticized in the literature.

The remainder of this paper is organized as follows. In Section 2, we briefly review the PFLRM and discuss the parameter estimation based on a regularized algorithm. In Section 3, the regularized PFLRM was identified and validated based on a data set collected from a $2.1 \mathrm{~kW}$ grid connected PV system during Jan. 12011 to June 30 2012. In Section 4, the prediction performance between the regularized PFLRM and some neural network models is compared. Some concluding remarks are given in Section 5.

\section{The Partial Functional Linear Regression Model}

\subsection{Classical functional linear model via functional principal components}

Functional principal component (FPC) analysis is a statistical method for investigating the dominant variation of functional data. Denote $x(t)$ as the square-integrable random 
function defined on a closed interval $T$ of the real line. Let $\mu(t)=E(x(t))$ and $G(s, t)=$ $\operatorname{cov}(x(s), x(t))$ be the mean and covariance of $x(t)$, respectively. The classic functional linear model is defined as

$$
y=\alpha+\int_{T}\{x(t)-\mu(t)\} \beta(t) d t+\varepsilon,
$$

where $\alpha$ is the intercept, the regression parameter function $\beta(t)$ is assumed to be squareintegrable, and $\varepsilon$ is a random error independent of $x(t)$. According to Mercer's theorem, the covariance function has the following spectral expansion:

$$
G(s, t)=\sum_{k=1}^{\infty} \lambda_{k} \phi_{k}(s) \phi_{k}(t),
$$

where $\lambda_{1} \geq \lambda_{2} \geq \ldots \geq 0$, and $\left\{\phi_{k}(t)\right\}$ is a complete orthonormal basis, which is usually called the FPC basis. Let $\left(x_{1},, y_{1}\right), \ldots,\left(x_{n}, y_{n}\right)$ be $n$ independent and identically distributed observation from $(x(t), y)$. The Karhunen-Loéve decomposition [29] forms the foundation of FPC analysis. By the Karhunen-Loéve theorem, one can express the centered process in the eigen-basis

$$
x_{i}(t)-\mu(t)=\sum_{k=1}^{\infty} \xi_{i k} \phi_{k}(t),
$$

where

$$
\xi_{i k}=\int_{T}\left\{x_{i}(t)-\mu(t)\right\} \phi_{k}(t) d t
$$

is the principal component associated with the $k$ th eigenfunction $\phi_{k}(t)$, which is also called the FPC score. The FPC scores are uncorrelated random variables with mean zero and variance

$$
\operatorname{Var}\left(\xi_{i k}\right)=E\left(\xi_{i k}^{2}\right)=\lambda_{k}
$$

7 Expanded on the orthonormal eigenbasis $\left\{\phi_{k}(t)\right\}$, the regression parameter function $\beta(t)$ becomes

$$
\beta(t)=\sum_{k=1}^{\infty} c_{k} \phi_{k}(t)
$$


where $c_{k}=\int_{T} \beta(t) \phi_{k}(t) d t$ are the basis coefficients. Then the functional linear model in Eq. (1) can be rewritten as

$$
y_{i}=\alpha+\sum_{k=1}^{\infty} c_{k} \xi_{i k}+\varepsilon_{i} .
$$

The other basis functions such as, B-spline, wavelet, and Fourier can also be used to approximate the regression parameter function $\beta(t)$ and the functional predictor $x_{i}(t)$. Compared with other basis functions, the FPC basis can represent the functional data in the most parsimonious way, in the sense that when using a fixed number of basis functions, the eigenfunction basis explains more variation than any other basis expansion. For this reason, we limit the discussion to FPC basis in this paper.

\subsection{The partial functional linear regression model with regularization}

To better predict 1-day ahead daily power output of the PV system, in addition to the valuable information about the 1-day ahead power output trajectories $x_{i}(t)$, some climatic variables can be included in the regression model. In this case, we can consider functional linear regression with functional predictor and scalar predictors. Suppose that the data are $(x(t), \mathbf{z}, y)$, where $\mathbf{z}=\left(z_{1}, \ldots, z_{p}\right)^{T}$ is a $p$-dimensional vector of scalar covariates. Let $\left(x_{i}, \mathbf{z}_{i}, y_{i}\right)_{i=1, \ldots, n}$ denote $n$ independent and identically distributed observations from the population $(x(t), \mathbf{z}, y)$. The response $y_{i}$, functional predictor $x_{i}$ and real predictor $\mathbf{z}_{i}$ can be centralized by subtracting them from their sample means. The sample means of $y_{i}, x_{i}$, and real predictor $\mathbf{z}_{i}$ are given by $\bar{x}=\frac{1}{n} \sum_{i=1}^{n} x_{i}, \bar{y}=\frac{1}{n} \sum_{i=1}^{n} y_{i}$, and $\overline{\mathbf{z}}=\frac{1}{n} \sum_{i=1}^{n} \mathbf{z}_{i}$, respectively. For the sake of simplicity, we use $y_{i}, x_{i}$ and $\mathbf{z}_{i}$ to represent the centralized data throughout the remaining of the paper. Based on the centralized data, we then model the linear relationship between $y_{i}$ and $\left(x_{i}, \mathbf{z}_{i}\right)$ by

$$
y_{i}=\int_{T} x_{i}(t) \beta(t) d t+\mathbf{z}_{i}^{T} \gamma+\varepsilon_{i},
$$

where $\gamma=\left(\gamma_{1}, \ldots, r_{p}\right)^{T}$ contains the regression coefficients of the non-functional covariates.

Expanding the functional predictor $x_{i}(t)$ and functional parameter $\beta(t)$ on the FPC basis $\phi_{k}$ as in the classical functional linear model (6), Eq. (7) can be written as

$$
y_{i}=\sum_{k=1}^{\infty} c_{k} \xi_{i k}+\mathbf{z}_{i}^{T} \gamma+\varepsilon_{i} .
$$


To estimate the coefficients $c_{k}$ of the regression parameter function and the coefficients $\gamma$, we consider the least-squares loss,

$$
L\left(c_{1}, c_{2}, \ldots, \gamma \mid\left(y_{1}, x_{1}, \mathbf{z}_{1}\right), \ldots,\left(y_{n}, x_{n}, \mathbf{z}_{n}\right)\right)=\sum_{i=1}^{n}\left(y_{i}-\sum_{k=1}^{\infty} c_{k} \xi_{i k}-\mathbf{z}_{i}^{T} \gamma\right)^{2}
$$

It is evident that the above loss function should not be directly minimized due to the infinite expansions of the functional predictor, requiring suitable regularization for $x$.

The conventional PFLRM selects the first few leading components to explain the majority of the total variation in the functional predictor $x(t)$. Assuming that $K$ leading components are selected, the response $y_{i}$ can be approximated by the truncated model

$$
y_{i} \cong \sum_{k=1}^{K} c_{k} \xi_{i k}+\mathbf{z}_{i}^{T} \gamma
$$

To estimate the above PFLRM, one needs to estimate the basis coefficients $\left(c_{1}, \ldots, c_{K}\right)$ and $\gamma$, which in turn requires the estimation of the unobserved FPC scores $\xi_{i k}$. Details of estimating $\xi_{i k}$ are shown in the Appendix.

After the FPC score estimate, $\hat{\xi}_{i k}$, was obtained, one can compute the estimates of $\left(c_{1}, \ldots, c_{K}\right)$ and $\gamma$ through minimizing

$$
\frac{1}{n} \sum_{i=1}^{n}\left(y_{i}-\hat{\boldsymbol{\xi}}_{i}^{T} \mathbf{C}-\mathbf{z}_{i}^{T} \boldsymbol{\gamma}\right)^{2}
$$

where $\hat{\boldsymbol{\xi}}_{i}=\left(\hat{\xi}_{i 1}, \ldots, \hat{\xi}_{i K}\right)^{T}$ and $\mathbf{C}=\left(c_{1}, \ldots, c_{k}\right)^{T}$. The estimate of functional parameter can then be computed based on $\hat{\beta}(t)=\sum_{k=1}^{K} \hat{c}_{k} \hat{\phi}_{k}(t)$.

Note that the value of $K$ in the conventional PFLRM is pre-specified or selected by explaining the percentage of total variance (usually $90 \%$ or $95 \%$ ) of $x$, or determined based on some model selection criteria such as the AIC criterion [27]. Despite its simplicity, this naive procedure of selecting $K$ could suffer from the following issues. First, the effect of FPC scores on the response does not necessarily coincide with their magnitudes specified by the autocovariance operator. For instance, some higher order FPC scores may contribute to the prediction of the model more than the leading FPC scores with lower orders. This phenomenon was first discussed by Hadi and Ling [31] in the principal component regression 
context and later was observed in real examples of high dimensional data [32] and functional data [33]. Second, this procedure may lead to the risk of overfitting, i.e., including insignificant components into the PFLRM, as pointed out by [28].

To accommodate the issues of selecting $K$ in the traditional PFLRM, one feasibility consists of using the regularized PFLRM. The key idea of it is to identify "important" components out of a sufficiently large number of candidates and to shrink those "unimportant" ones to zero. Least absolute shrinkage and selection operator (Lasso) for variable selection has been widely discussed over the past decades [34]-[38]. Some recent variations include the grouped Lasso [39] and the adaptive Lasso ([36, 40]. In the present paper, we consider the estimate of PFLRM based on the Lasso penalty. In particular, the regularized PFLRM can be estimated by minimizing

$$
\frac{1}{n} \sum_{i=1}^{n}\left(y_{i}-\hat{\boldsymbol{\xi}}_{i}^{T} \mathbf{C}-\mathbf{z}_{i}^{T} \boldsymbol{\gamma}\right)^{2}+\tau\left(\sum_{k=1}^{K}\left|c_{k}\right|+\sum_{j=1}^{p}\left|\gamma_{j}\right|\right),
$$

where $\tau>0$ is the regularization parameter. Different criteria such as such as crossvalidation $(\mathrm{CV})$, generalized cross-validation(GCV), $\mathrm{AIC}$ and $\mathrm{BIC}$, can be used to select the regularization parameter $\tau$. In this paper, we used BIC to choose $\tau$.

\section{Model Identification}

The data used for the present study is based on the data recorded from a $2.1 \mathrm{~kW}$ grid connected PV system. The underlying PV system is located in the Coloane island of Macau Special Administrative Region (SAR) (latitude $=22^{\circ} 10^{\prime} 0^{\prime \prime} N$ and longitude $=113^{\circ} 33^{\prime} 0^{\prime \prime} E$ ). It consists of 12 Kyocera HTS-175 $(1.29 \mathrm{~m} \times 0.99 \mathrm{~m})$ with an installed capacity of $2.1 \mathrm{~kW}$ and an inclination angle of $10^{\circ}$, facing south-east. Under standard test conditions (i.e., cell temperature $=25^{\circ} \mathrm{C}$ ), the maximum output power of the HTS-175 module was $175 \mathrm{~W} \pm 5 \%$. A single Xantrex ${ }^{T M}$ grid tie (GT series) solar inverter was used to convert direct current (DC) to alternating current (AC). Under AC output voltage of $240 \mathrm{~V}$, this inverter can provide maximum output current of $11.7 \mathrm{~A}$. The maximum AC power output is $2.8 \mathrm{~kW}$ for the electrical input. This inverter has MPPT operating range from $193 \mathrm{Vdc}$ to $550 \mathrm{Vdc}$ with the maximum input current of 15.4 Adc. The maximum inverter efficiency is $95 \%$. 


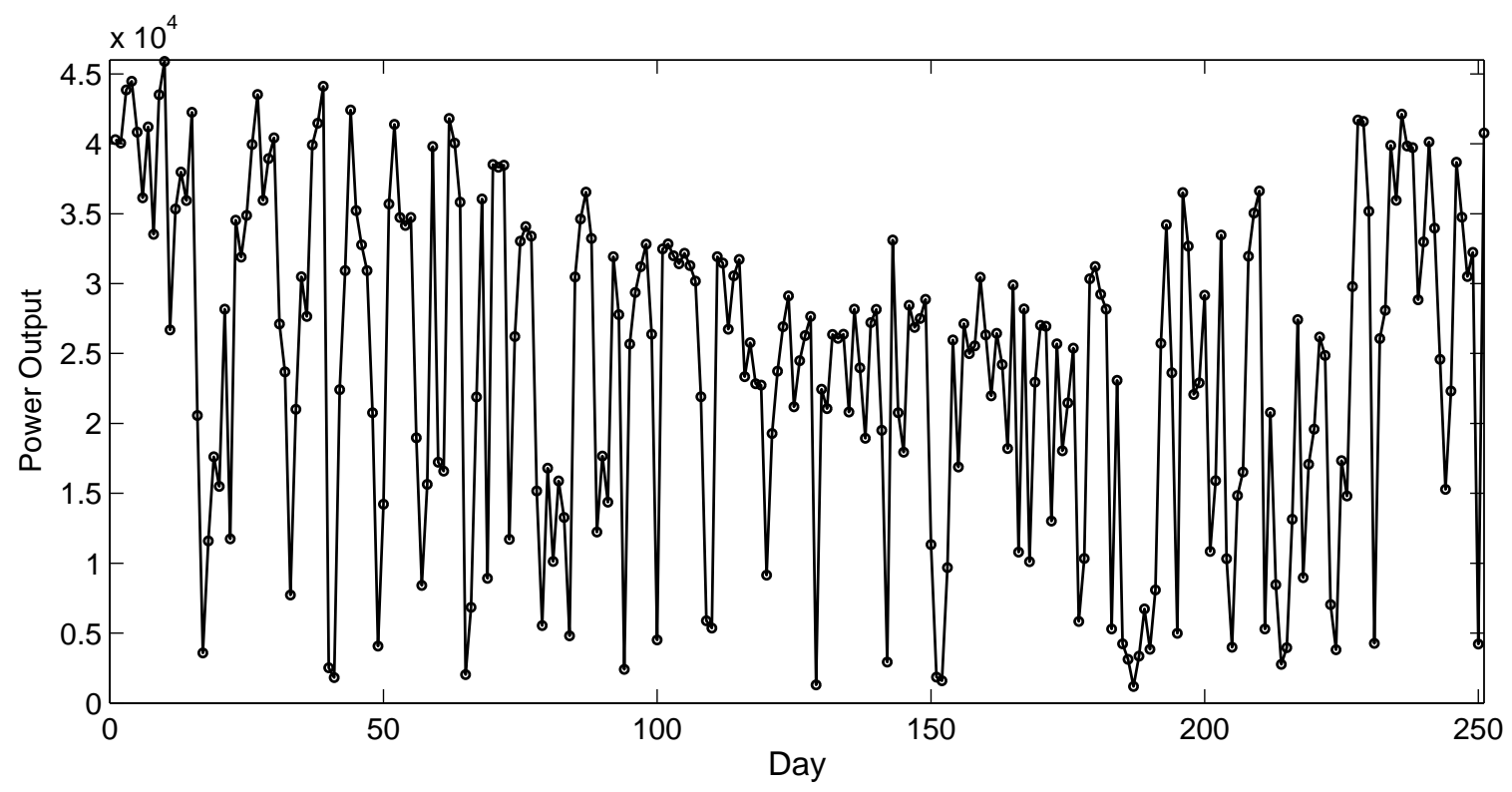

Figure 1: Mean daily power output in 2011.

The data during the period from January 1, 2011 to June 30, 2012 were recorded. The measurements on array power output and global horizontal irradiance(GHI) were recorded at 1-min sampling intervals. In the study, we use the records during the period from January 1 to December 312011 as the training data, and the remaining data from January 1 to June 302012 as testing data. The underlying system automatically turns on at 6 am and off at $7 \mathrm{pm}$ each day. It is inevitable that there are some missing data due to maintenance or instrument malfunction. After removing the data with missing values and outliers, there are 465 daily records in the present data set, including 321 training data records and 144 testing data records. Fig. 1 depicts the mean daily output power generated from the PV system during the period from January 1 to December 31, 2011. As can be seen from Fig. 1 , the mean daily power output varies from $60 \mathrm{~W}$ to $1,000 \mathrm{~W}$.

To estimate the PFLRM and regularized PFLRM, we used the 1-day ahead power output trajectory as the functional predictor in Eq. (8). For this reason, the observation time and the recorded power output of the $j$ th measurement in day $i-1$ will be denoted as $t_{i j}$ and $U_{i j}$, respectively. The power output is observed at 1-min interval during 6:00 am to 6:59 pm every day. There are 780 minutely observations each day. When it is in the early morning 
Table 1: Summary statistics of the meteorological variables

\begin{tabular}{cccccccc}
\hline Variable(Variable symbol, Unit) & Min & Q1 & Median & Q3 & Max & Mean & Variance \\
\hline Pressure $\left(z_{1}, P a\right)$ & 995.2 & 1007.2 & 1012.5 & 1017.8 & 1025.9 & 1012.2 & 44.6 \\
Max Temperature $\left(z_{2},{ }^{o} C\right)$ & 7.4 & 19.9 & 26.5 & 29.5 & 36.1 & 24.8 & 39.10 \\
Mean Temperature $\left(z_{3},{ }^{o} C\right)$ & 6.1 & 16.6 & 23.0 & 26.6 & 31.4 & 21.5 & 34.7 \\
Min Temperature $\left(z_{4},{ }^{\circ} C\right)$ & 5.0 & 14.0 & 20.6 & 24.6 & 28.6 & 19.4 & 34.4 \\
Dew Temperature $\left(z_{5},{ }^{o} C\right)$ & -1.0 & 13.2 & 19.1 & 23.3 & 24.9 & 17.8 & 36.2 \\
Humidity $\left(z_{6}, R H\right)$ & 41.0 & 75.0 & 81.0 & 88.0 & 98.0 & 80.5 & 99.5 \\
Insolation $\left(z_{7}, l u x\right)$ & 0 & 0 & 2.8 & 7.2 & 12.5 & 3.9 & 14.6 \\
Wind speed $\left(z_{8}, m / s\right)$ & 5.0 & 9.0 & 12.0 & 16.0 & 44.0 & 13.4 & 38.2 \\
Precipitation $\left(z_{9}, m l\right)$ & 0 & 0 & 0.01 & 1.2 & 92.8 & 3.9 & 117.1 \\
\hline
\end{tabular}

(near 6:00 am) or in the late afternoon (near 6:59 pm), the recorded power output $U_{i j}$ is less than or equal to zero as there is no power output generated at that time. Within each day, such observations would be discarded when estimating the PFLRM and regularized PFLRM. With the removal of $U_{i j} \leq 0$, the number of observations used to estimate the PFLRM would vary from day to day. Based on the training data set $\left\{\left(t_{i j}, U_{i j}\right), 1 \leq i \leq 321\right\}$, we can obtain the estimate of FPC score $\hat{\xi}_{i k}$, details of which are shown in the Appendix. The "PACE" package in Matlab was used to implement the algorithm for estimating the FPC scores, which is available from the website http://www.utstat.utoronto.ca/fyao/.

Similar to [20], we also include some meteorological variables in the prediction model to improve forecast accuracy. Following [20], the following meteorological variables that can be easily obtained from the local observatory are considered: mean atmospheric pressure $\left(z_{1}\right)$, highest temperature $\left(z_{2}\right)$, average temperature $\left(z_{3}\right)$, lowest temperature $\left(z_{4}\right)$, dew temperature $\left(z_{5}\right)$, humidity $\left(z_{6}\right)$, insolation $\left(z_{7}\right)$, average wind speed $\left(z_{8}\right)$, and precipitation $\left(z_{9}\right)$. The local observatory, Macao Meteorological and Geophysical Bureau, is located in Taipa island of Macau SAR with latitude $=22^{\circ} 09^{\prime} 36^{\prime \prime} N$ and longitude $=113^{\circ} 33^{\prime} 54^{\prime \prime} E$. The distance from the underlying grid-connected PV system to the local observatory is around $1.7 \mathrm{~km}$. Table 1 lists the summary statistics of these meteorological variables, including minium, the 
Table 2: Parameter estimates of the PFLRM and regularized PFLRM for different values of $K$

\begin{tabular}{|c|c|c|c|c|c|}
\hline \multirow[b]{2}{*}{ Parameter } & \multicolumn{2}{|c|}{$K=2$} & \multicolumn{2}{|c|}{$K=7$} & \multirow[b]{2}{*}{ MLR } \\
\hline & PFLRM & RPFLRM & PFLRM & RPFLRM & \\
\hline $\operatorname{Intercept}(\alpha)$ & -596.8497 & -1332.90 & -1033.34 & -1067.30 & 4006.74 \\
\hline $\operatorname{pressure}\left(z_{1}\right)$ & 0.9629 & 1.711 & 2.10 & 1.45 & -3.76 \\
\hline Max Temperature $\left(z_{2}\right)$ & 5.2117 & 0 & 4.62 & 0 & 9.38 \\
\hline mean Temperature $\left(z_{3}\right)$ & -13.2566 & 0 & -43.51 & 0 & -34.14 \\
\hline Min Temperature $\left(z_{4}\right)$ & -2.6986 & 0 & 3.90 & 0 & 49.60 \\
\hline Dew Temperature $\left(z_{5}\right)$ & 14.0358 & 0 & 36.74 & 0 & -34.47 \\
\hline $\operatorname{Humidity}\left(z_{6}\right)$ & -3.7665 & 0 & -7.63 & 0 & 6.17 \\
\hline $\operatorname{Insolation}\left(z_{7}\right)$ & 5.1346 & 1.70 & 3.02 & 1.87 & -6.04 \\
\hline Wind $\operatorname{speed}\left(z_{8}\right)$ & -0.3225 & 0 & 0.97 & 0 & 1.55 \\
\hline Precipation $\left(z_{9}\right)$ & -0.3002 & 0 & 0.06 & 0 & 1.03 \\
\hline 1st FPC scores $\left(\hat{\xi}_{1}\right)$ & -0.0044 & 0.16 & 0.15 & 0.15 & - \\
\hline 2nd FPC scores $\left(\hat{\xi}_{2}\right)$ & -0.0230 & 0 & -0.00 & 0 & - \\
\hline 3 th FPC scores $\left(\hat{\xi}_{3}\right)$ & - & - & -0.06 & -0.06 & - \\
\hline 4th FPC scores $\left(\hat{\xi}_{4}\right)$ & - & - & 3.69 & 3.29 & - \\
\hline 5 th FPC scores $\left(\hat{\xi}_{5}\right)$ & - & - & -2.98 & 0 & - \\
\hline 6th FPC scores $\left(\hat{\xi}_{6}\right)$ & - & - & -17.33 & 0 & - \\
\hline 7th FPC scores $\left(\hat{\xi}_{7}\right)$ & - & - & 8.78 & 0 & - \\
\hline
\end{tabular}

Note: RPFLRM represents regularized PFLRM, and MLR represents the multiple linear regression model. 
first quartile (Q1), median, the third quartile (Q3), maximum, mean, and variance.

After obtaining the estimates of FPC scores $\hat{\xi}_{i k}$ and the inclusion of the above nine climatic variables in the regression model, the conventional PFLRM can be estimated based on minimizing Eq. (11) for a prespecified number of leading principal components, $K$. To estimate the regularized PFLRM, the "LASSO" package in Matlab can be used to optimize Eq. (12). In summary, the procedure for estimating PFLRM and regularized PFLRM consists of the following steps:

(i) Organize the data set for estimating the FPC scores, $\left\{\left(t_{i j}, U_{i j}\right)\right\}$. As the 1-day ahead power output trajectory was treated as the functional predictor, we set $U_{i j}$ as the $j$ th measured power output in day $i-1$, and the corresponding observation time is recorded as $t_{i j}$. When $i=1$, we just set $U_{1 j}$ and $t_{1 j}$ to be the $j$ th observed power output and observation time in the first day respectively for the sake of simplicity;

(ii) Obtain the FPC score estimates for each day from the paired observations $\left(t_{i j}, U_{i j}\right)$ by using the "PACE" package in Matlab, $\hat{\boldsymbol{\xi}}_{i}=\left(\hat{\xi}_{i 1}, \ldots, \hat{\xi}_{i K}\right)^{T}$;

(iii) Estimate the PFLRM for a fixed value of $K$. Set $y_{i}$ as the measured daily average power output in day $i$, and organize the data $\left(y_{i}, \hat{\boldsymbol{\xi}}_{i}, \mathbf{z}_{i}\right)$ in matrix format $\left[\begin{array}{lll}y_{i} & \hat{\boldsymbol{\xi}}_{i}^{T} & \mathbf{z}_{i}^{T}\end{array}\right]$. The optimal parameters to Eq. (11) can be obtained by performing the conventional least-squares regression of $y$ on the predictors $\left[\hat{\boldsymbol{\xi}}^{T} \mathbf{z}^{T}\right]$;

(iv) Estimate the regularized PFLRM in Eq. (12) based on the LASSO algorithm in Matlab. BIC criterion was used to choose the regularization parameter.

Table 2 lists the parameter estimates of the PFLRM and regularized PFLRM together with the multiple linear regression (MLR) estimates. Two different values of $K$ ranging from small to large are considered, including $K=2$ and 7 . From Table 2, the regularized PFLRM is simpler than the PFLRM to some extent as expected. This is because the regularized PFLRM can automatically shrink some unimportant FPC scores and insignificant explanatory variables to zero in the prediction model. For example, when $K=2$, the regularized 
PFLRM only includes the first FPC score and explanatory variables $z_{1}$ (Mean atmospheric pressure) and $z_{7}$ (Insolation) in the model. Instead, the conventional PFLRM includes the first two FPC scores and all the 9 explanatory variables. This is even obvious when $K$ is relatively large. It can be seen that when $K=7$, the regularized PFLRM only includes the first, second, and fourth FPC scores while the conventional PFLRM includes all the first 7 FPC scores except the second.

As compared to the multiple linear regression model, the regularized PFLRM seems to have better interpretability. As can be seen from Table 2 , the regression coefficients on $z_{7}$ (Insolation) is positive in the regularized PFLRM for both $K=2$ and $K=7$. This is consistent with the intuition that the higher the insolation is, the higher power output tends to be generated. However, the regression coefficient on $z_{7}$ (Insolation) in the multiple linear regression model is negative (-6.04), which causes certain difficulty in interpretation. One partial reason is due to collinearity among the climatic variables, $z_{1}, \ldots, z_{9}$.

To measure the goodness-of-fit of the PFLRM, three measures of performance are considered: the root of mean square errors (RMSE), mean absolute deviation (MAD), and mean absolute percent error (MAPE). The RMSE, MAD, and MAPE are computed with

$$
\begin{gathered}
R M S E=\sqrt{\frac{\sum_{i=1}^{n}\left(y_{i}-\hat{y}_{i}\right)^{2}}{n},} \\
M A D=\frac{\sum_{i=1}^{n}\left|y_{i}-\hat{y}_{i}\right|}{n},
\end{gathered}
$$

and

$$
M A P E=\frac{\sum_{i=1}^{n}\left|y_{i}-\hat{y}_{i}\right| / y_{i}}{n}
$$

respectively, where $\hat{y}_{i}$ is the predict value. For the PFLRM and regularized PFLRM,

$$
\hat{y}_{i}=\sum_{k=1}^{K} \hat{c}_{k} \hat{\xi}_{i k}+\mathbf{z}_{i}^{T} \hat{\gamma},
$$

where $\hat{c}_{1}, \ldots, \hat{c}_{k}$ are the fitted basis coefficients, and $\hat{\gamma}$ is the vector of fitted regression coefficients. 
Table 3: The RMSE, MAD, MAPE values of the MLR, PFLRM, and regularized PFLRM based on the training data

\begin{tabular}{ccccc}
\hline$K$ & Methods & RMSE & MAD & MAPE \\
\hline & MLR & 206.9397 & 172.6356 & 0.5679 \\
2 & PFLRM & 62.3022 & 34.5456 & 0.0848 \\
& RPFLRM & 63.1742 & 35.0702 & 0.0886 \\
7 & PFLRM & 62.4148 & 35.1270 & 0.0877 \\
& RPFLRM & 63.3948 & 35.6129 & 0.0920 \\
\hline
\end{tabular}

Table 3 summarizes the RMSE, MAD, and MAPE values of the PFLRM, regularized PFLRM, and multiple linear regression for the training data. As can be seen from Table 3, the RMSE, MAD, and MAPE values of PFLRM and the regularized PFLRM are much smaller than those of multiple linear regression model. Therefore, the PFLRM and regularized PFLRM that make use of the intra-day trajectory of power output can greatly improve the model accuracy over the multiple linear regression model as expected. Moreover, it can be observed from Table 3 that the PFLRM tends to have smaller RMSE, MAD, and MAPE values than the regularized PFLRM. However, the difference is slight. The slightly better fit of the PFLRM is due to the inclusion of the additional variable and FPC components in the regression model, as compared to the regularized PFLRM. In view that the regularized PFLRM has very competitive prediction performance to the PFLRM but achieves great simplicity in model format, we suggest it for practical use. Throughout the remaining of this paper, we limit discussion to the regularized PFLRM.

Fig. 2 further plots the estimated mean function, the first two estimated eigenfunctions, and estimated functional coefficients of the regularized PFLRM with $K=2$. From Fig. 2(c), we can see that the functional parameter estimate reaches its maximum at 12 am, which indicates that the observation at this point is very influential to the prediction of the next-day power output. The QQ plot of the residuals from the regularized PFLRM with $K=2$ is shown in Fig. 3, which provides a visual aid to check if the residuals are 
normally distributed. As can be seen from Fig. 3, the residuals approximately fit a straight line, implying that the residuals can be approximately assumed to be normal. That is, it is reasonable to assume that the model error $\varepsilon$ in Eq. (7) is close to normal, and that the fitted regularized PFLRM is appropriate.

\section{Comparison with Neural Network Models}

Among the various approaches to forecasting power output, the neural network model seems to be the dominating approach. It is of interest here to compare the prediction performance between the neural network models and the regularized PFLRM. In particular, two neural network models are considered, including radial basis function (RBF) and multilayer perception (MLP) neural networks. Both MLP and RBF are supervised learning techniques, and are feed-forward artificial neural networks that map sets of input data onto a set of appropriate outputs. Generally speaking, MLP can find more complex relationship than RBF, and RBF is faster than MLP.

The RBF neural networks usually consist of three layers: input layer, hidden layer, and output layer. The nodes within each layer are connected to the previous layer. The input layer is simply a set of sensory units. The second layer, hidden layer, contains a nonlinear activation function to perform a transformation from the input space to a higher-dimension hidden-unit space. The RBF network often uses the radial basis function (RBF) or the Gaussian soft-max function as the activation function, which is analogous to the sigmoid function. The output of the neural network model is associated with the hidden units based on a weighted sum, given by

$$
\hat{y}=\sum_{k=1}^{m} \omega_{i} \phi(\cdot),
$$

where $m$ is the number of neurons in the hidden layer, $\omega_{i}$ is the weight between the hidden and output layer, and $\phi(\cdot)$ is the activation function.

Compared to the RBF neural network with one hidden layer, the MLP network can be designed with more than one hidden layer. The use of many such hidden layers is very useful for deep learning. Neurons of $i$-th layer serve as input features for neurons of $i+1$ th layer. 


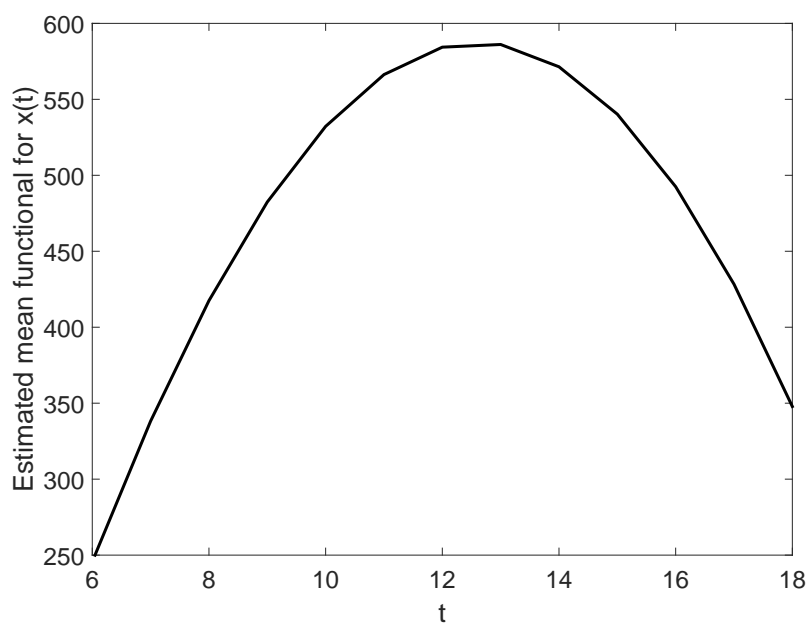

(a)

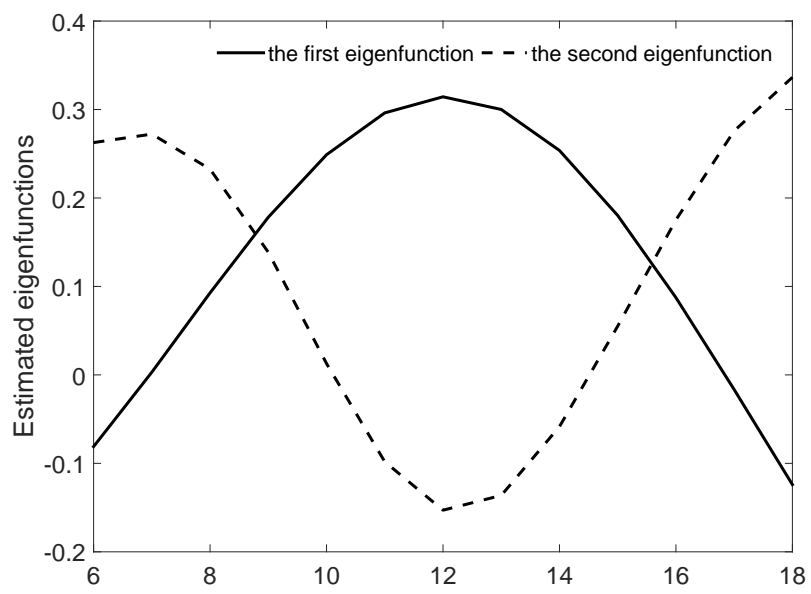

(b)

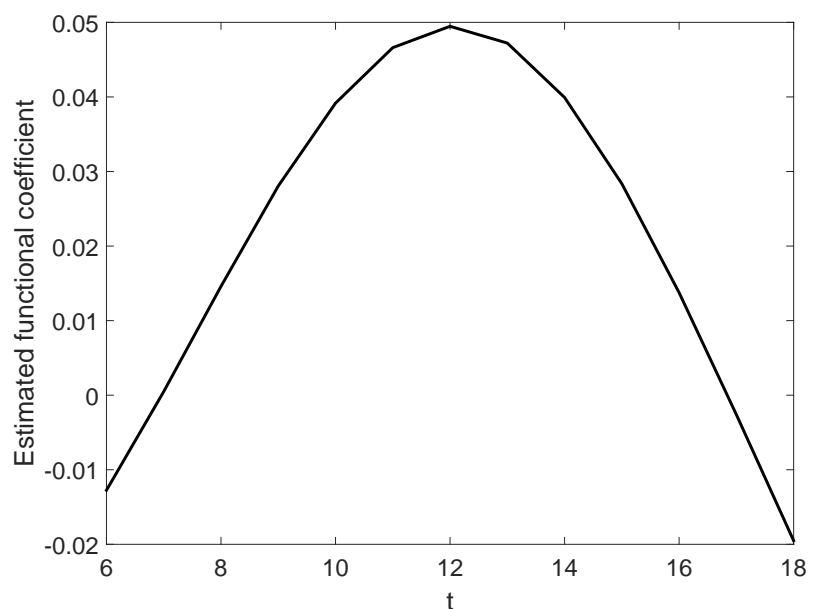

(c)

Figure 2: Estimates of mean function $\mu(t)$ for functional predictor $x(t)$ (a), the first two eigenfunctions (b), and the functional coefficient (c) of the regularized PFLRM $(K=2)$. 


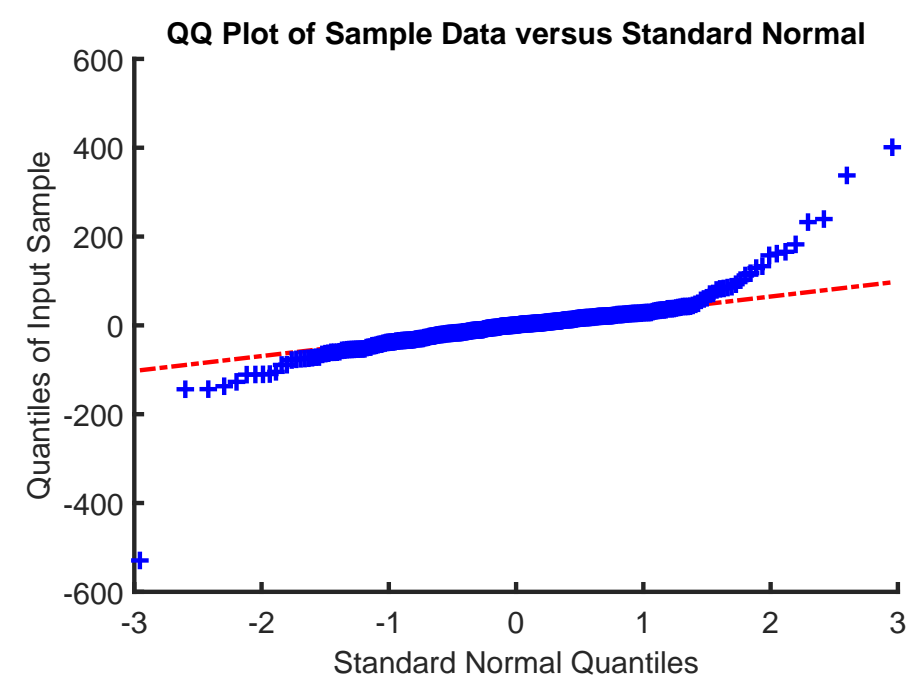

Figure 3: QQ plot of the residuals of the regularized PFLRM $(K=2)$.

There are connection weights between each neuron. Output of hidden neuros are converted to nonlinear values based on an activation function. The two main activation functions used in current applications for MLP are given by

$$
f\left(v_{i}\right)=\tanh \left(v_{i}\right) \text {, and } g\left(v_{i}\right)=\left(1+e^{-v_{i}}\right)^{-1} \text {, }
$$

which are both sigmoid. The MLP neural networks learn using an algorithm called backpropagation. With backpropagation, the input data is repeatedly presented to the neural network. With each presentation the output of the neural network is compared to the desired output and an error is computed. This error is then feed back (backpropagated) to the neural network and used to adjust the weights such that the error decreases with each iteration and the neural model gets closer and closer to producing the desired output.

Table 4 summarizes the RMSE, MAD, and MAPE values of the regularized PFLRM and the neural network models based on the training data set. In the neural network models, the input variables consist of all the nine climatic variables, $z_{1}, z_{2}, \ldots, z_{9}$, and the first two or seven FPC scores of the functional predictor. The parameter estimates of the neural network models were obtained based on software SPSS 18. The RBF model used 10 and 9 neuros in the hidden layer for $K=2$ and $K=7$, respectively, and the activation function used is based on Softmax. The MLP model was designed with one hidden layer with 6 neuros, and 
Table 4: The RMSE, MAD, and MAPE values of the MLP, RBF, and regularized PFLRM based on the training sample

\begin{tabular}{ccccc}
\hline$K$ & Methods & RMSE & MAD & MAPE \\
\hline$K=2$ & MLP & 64.0621 & 41.8335 & 0.1177 \\
& RBF & 127.3511 & 98.7994 & 0.2914 \\
& RPFLRM & 63.1742 & 35.0702 & 0.0886 \\
\hline \multirow{2}{*}{$K=7$} & MLP & 59.8273 & 39.0352 & 0.1103 \\
& RBF & 121.4909 & 97.7094 & 0.2947 \\
& RPFLRM & 63.3948 & 35.6129 & 0.0920 \\
\hline
\end{tabular}

the activation function is based on hyperbolic trigonometric. As can be seen from Table 4, when $K=2$, the regularized PFLRM has smaller RMSE, MAD, and MAPE than the MLP model, and than the RBF model. When $K=7$, the MLP model gives the smallest RMSE, followed by the regularized PFLRM, and the RBF model.

Table 5 summarizes the RMSE, MAD, and MAPE values of the regularized PFLRM and the neural network models applied to the testing data set. It can be seen from Table 5 that the regularized PFLRM has the best prediction performance for both cases with $K=2$ and $K=7$. For example, when $K=2$, the RMSE value of the regularized PFLRM is 59.17, which is less than that of MLP (74.97) and RBF (180.65). The MAPE of the regularized PFLRM is $11.34 \%$, which is also less than the corresponding value of $20.92 \%$ for MLP and that of $63.88 \%$ for RBF. In addition to the better prediction performance, it is important to note that the regularized PFLRM enjoys certain simplicity in model identification as compared to the neural network models as it has fewer parameters to estimate.

Figure 4 further plots the measured and forecasted daily power output based on the MLP, RBF, and the regularized PFLRM for the first six months in 2012. The comparison results are similar under the cases with $K=2$ and $K=7$, here we only plot the result under the case with $K=2$ for the sake of simplicity. As can be seen from Figure 4 , the 

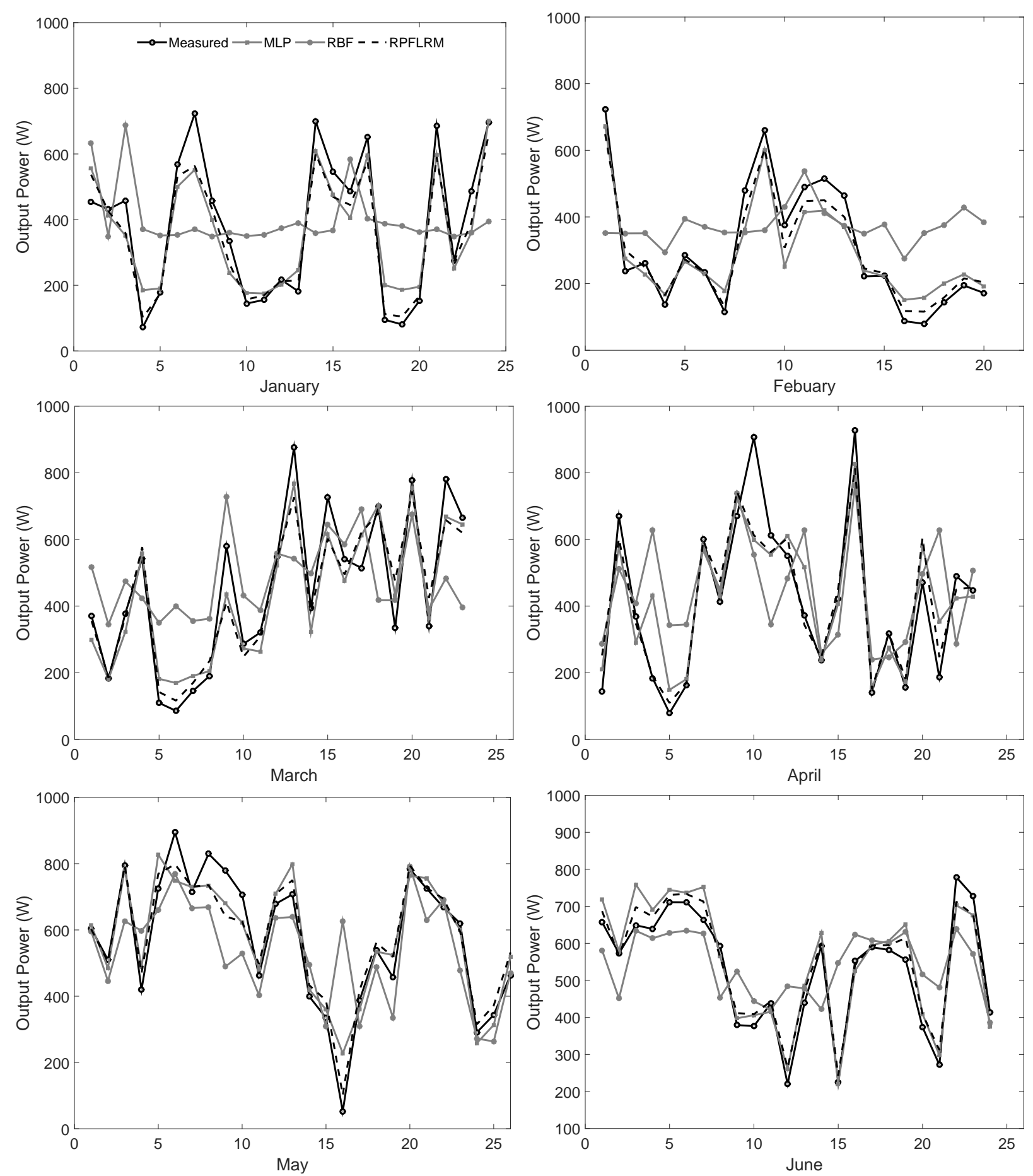

Figure 4: Comparison between the 1-day ahead forecast and measured values of power output of the PV system during January to June 2012 among the MLP, RBF, and regularized PFLRM based on $K=2$. 
Table 5: The RMSE, MAD, and MAPE values of the MLP, RBF, and regularized PFLRM based on the testing sample

\begin{tabular}{ccccc}
\hline$K$ & Methods & RMSE & MAD & MAPE \\
\hline$K=2$ & MLP & 74.9716 & 57.9138 & 0.2092 \\
& RBF & 180.6523 & 147.3396 & 0.6388 \\
& RPFLRM & 59.1704 & 40.6277 & 0.1134 \\
\hline \multirow{2}{*}{$K=7$} & MLP & 76.5793 & 57.8320 & 0.1886 \\
& RBF & 177.2916 & 145.9263 & 0.6683 \\
& RPFLRM & 59.3899 & 40.4519 & 0.1204 \\
\hline
\end{tabular}

forecast of daily power output based on the regularized PFLRM seems to be the closest to the measured value on average among all the methods considered here, although it may not produce the smallest forecast error at each time point. Also we can see that RBF has much larger forecast error than MLP on average.

\section{Conclusion}

With recent advancements in the sensor and data acquisition technology, the solar data is often measured on a minute interval. The minutely data is more informative than the aggregated mean daily solar data. However, the conventional forecasting methods such as multiple linear regression and neural network models cannot make use of the valuable information from the minutely solar data when forecasting the mean daily output power. Instead, they can only use the historical aggregated solar data to generate the 1-day ahead forecast. The use of aggregated daily solar data for forecasting the mean daily power output of PV system could cause certain a great loss of information in forecasting, which in turn affects forecasting accuracy. Aimed at incorporating the trajectory of power output into forecasting, this paper suggests a PFLRM for the 1-day ahead forecasting of the mean daily output power of a $2.1 \mathrm{~kW}$ grid connected PV system. A regularized algorithm was used to 
estimate the parameters.

The comparison results show that the regularized PFLRM improves the forecast accuracy of the multiple linear regression and neural network models. In the regularized PFLRM model, it is shown that the information about the climatic variables such as mean atmospheric pressure and insolation, and the first FPC score of the functional predictor are valuable in forecasting the power output of a PV system. In addition to the better prediction performance, the regularized PFLRM gets away from the requirement of past experience and reliable knowledge, unlike the neural network models. Therefore, the regularized PFLRM is much simpler than the neural network models for forecasting.

Although the analysis is based on the real data set collected in Macau, the proposed model can be easily applied in other locations with different climate regime. The climatic variables considered may be different for different climate regime. The proposed regularized PFLRM can automatically select the important variables that significantly affect the response variable. The proposed method can be also extended in other respects. First, this paper mainly focuses on the direct approach to the forecast power output of PV systems. It is straightforward to extend it to the indirect approach, i.e., to first forecast the solar irradiance and then to forecast the power output based on the forecasted solar irradiance information. Second, it is also possible to consider some of the important time-varying meteorological variables as functional predictors but not as covariates. For example, one can also consider the time-varying pressure and insolation as functional predictors in the regularized PFLRM. This needs more investigation in the future.

\section{Appendix: Estimate of FPC Scores}

To estimate the FPC scores, one needs to first estimate the mean function $\mu(t)$ and covariance function $G(s, t)$. In practice we do not observe the entire trajectories $x_{i}(t)$, but only have intermittent noisy measurements due to measurement errors or other noisy variables. Let $x_{1}, \ldots, x_{n}$ as defined before. For each $i$, we observe pairs $\left(t_{i j}, U_{i j}\right)$ for $1 \leq$ 
$j \leq L_{i}$, where

$$
U_{i j}=x_{i}\left(t_{i j}\right)+e_{i j}=\mu\left(t_{i j}\right)+\sum_{k=1}^{\infty} \xi_{i k} \phi_{k}\left(t_{i j}\right)+e_{i j}, \quad 1 \leq i \leq n .
$$

The observation times $t_{i j}$ all lie in the compact interval $T$, and each $L_{i} \geq 2$, which represents the repeated number of independent observations collected for each $i$. Here the errors $e_{i j}$ are assumed to be white noise with zero mean and constant variance, which are independent of the FPC scores. These assumptions are not very restricted and are commonly made in the literature. With these assumptions, the FPC score $\xi_{i k}$ can be estimated based on a linear form.

Using the data set $\left\{\left(t_{i j}, U_{i j}\right), 1 \leq i \leq n, 1 \leq j \leq L_{i}\right\}$, we wish to construct estimators $\hat{\mu}(t)$ and $\hat{K}(s, t)$. By using $G_{i}\left(t_{i j}, t_{i l}\right)=U_{i j} U_{i l}$, one can obtain the estimates, $\hat{\mu}(t)$ and $\hat{G}(s, t)$ based on local linear or surface smoother through minimizing the following two functions:

$$
\sum_{i=1}^{n} \sum_{j=1}^{L_{i}} \kappa_{1}\left(\frac{t_{i l-t}}{h_{1}}\right)\left(U_{i j}-a_{0}-a_{1}\left(t-t_{i j}\right)\right)^{2}
$$

and

$$
\sum_{i=1}^{n} \sum_{1 \leq l \neq j \leq L_{i}} \kappa_{2}\left(\frac{t_{i l}-s}{h_{x}}, \frac{t_{i j}-t}{h_{x}}\right)\left\{G_{i}\left(t_{i l}, t_{i j}\right)-f\left(\eta,(s, t),\left(s_{i l}, s_{i j}\right)\right)\right\}^{2},
$$

where $\kappa_{1}$ and $\kappa_{2}$ are the kernel functions with one or two dimensions, and $\left.f\left(\eta,(s, t),\left(t_{i l}, t_{i j}\right)\right)\right)=$ $\eta_{0}+\eta_{11}\left(s-t_{i l}\right)+\eta_{12}\left(t-t_{i j}\right)$. Denote $\left(\hat{a}_{0}(t), \hat{a}_{1}(t)\right)$ and $\left(\hat{\eta}_{0, x}(s, t), \hat{\eta}_{11, x}(s, t), \hat{\eta}_{12, x}(s, t)\right)$ as the optimal solutions to Eqs. (20) and (21) respectively, then the estimates of $\mu(t)$ and $G(s, t)$ are given by

$$
\hat{\mu}(t)=\hat{a}_{0}(t)
$$

and

$$
\hat{G}(s, t)=\hat{\eta}_{0, x}(s, t),
$$

respectively.

After obtaining the estimate $\hat{G}(s, t)$, eigenfunctions and eigenvalues can then be estimated by solving the eigen-equation

$$
\int_{T} \hat{G}(s, t) \hat{\phi}_{k}(s) d s=\hat{\lambda}_{k} \hat{\phi}_{k}(t)
$$


subject to $\int_{T} \hat{\phi}_{k}(t)^{2} d t=1$ and $\int_{T} \hat{\phi}_{k}(t) \hat{\phi}_{m}(t) d t=0$ for $m<k$. The constraint $\int_{T} \hat{\phi}_{i}(t)^{2} d t=1$ guarantees that the norm of the estimated eigenfunction $\hat{\phi}_{i}(t)$ is one while the constraint $\int_{T} \hat{\phi}_{k}(t) \hat{\phi}_{m}(t) d t=0$ guarantees that the eigenfunctions are mutually orthogonal.

Assuming that $\xi_{i k}$ and $e_{i l}$ are jointly Gaussian in Eq. (19), the best linear prediction of the FPC score for the $i$ th subject is given by

$$
\tilde{\xi}_{i k}=E\left[\xi_{i k} \mid \mathbf{U}_{i}\right]=\lambda_{k} \boldsymbol{\phi}_{i k}^{T} \boldsymbol{\Sigma}_{U_{i}}^{-1}\left(\mathbf{U}_{i}-\boldsymbol{\mu}_{i}\right),
$$

where $\mathbf{U}_{i}=\left(U_{i 1}, \ldots, U_{i L_{i}}\right)^{T}, \boldsymbol{\mu}_{i}=\left(\mu\left(t_{i 1}\right), \ldots, \mu\left(t_{i L_{i}}\right)\right)^{T}$, and $\phi_{i k}=\left(\phi_{k}\left(t_{i 1}\right), \ldots, \phi_{k}\left(t_{i L_{i}}\right)\right)^{T}$. The element in row $j$ and column $l$ of matrix $\Sigma_{U_{i}}$ is given by $\left(\Sigma_{U_{i}}\right)_{j l}=G\left(t_{i j}, t_{i l}\right)+\tilde{\sigma}^{2} \delta_{j l}$, where $\delta_{j l}=1$ if $j=l$ and 0 if $j \neq l$. Replacing $\boldsymbol{\mu}_{i}$ in Eq. (25) with its estimate, one can obtain the estimate of FPC score as

$$
\hat{\xi}_{i k}=\hat{E}\left[\xi_{i k} \mid \mathbf{U}_{i}\right]=\hat{\lambda}_{k} \hat{\phi}_{i k}^{T} \hat{\Sigma}_{U_{i}}^{-1}\left(\mathbf{U}_{i}-\hat{\boldsymbol{\mu}}_{i}\right) .
$$

For more detailed discussions about the estimate of FPC scores, one can refer to [27] and [30]. To estimate the mean function of $\mu(t)$ and the covariance function $G(s, t)$, we can also use other smoothing methods such as smoothing spline or penalized spline. However, when smoothing spline or penalized spline is used, a mixture model is required. Therefore, the resulting computational cost is high as compared to the local linear or surface smoothing.

\section{References}

[1] Strzalka A, Alam N, Duminil E, Coors V, Eicker U. Large scale integration of photovoltaics in cities. Appl Energy 2012; 93:413-421.

[2] Woyte A, Thong VV, Belmans R, Nijs J. Voltage fluctuations on distribution level introduced by photovoltaic systems. IEEE Trans Energy Convers 2006; 21: 202-209.

[3] Pedro HTC, Coimbra CFM. Assessment of forecasting techniques for solar power production with no exogenous inputs. Solar Energy 2012; 86:2017-2028.

[4] Lopez ML, Cardona MS. Multiplicative ARMA models to generate hourly series of global irradiation. Solar Energy 1998; 63:283-291.

[5] Martín L, Zarzalejo LF, Polo J, Navarro A, Marchante R, Cony M. Prediction of global solar irradiance based on time series analysis: Application to solar thermal power plants energy production planning. Solar Energy 2010; 84:1772-1781. 
[6] Reikard G. Predicting solar radiation at high resolutions: A comparison of time series forecasts. Solar Energy 2009; 83:342-349.

[7] Sfetsos A, Coonick A. Univariate and multivariate forecasting of hourly solar radiation with artificial intelligence techniques. Solar Energy 2000;68(2):169-178.

[8] Kalogirou SA. Artificial neural networks in renewable energy systems applications: a review. Renew Sustain Energy Rev 2001; 5:373-401.

[9] Dorvlo SS, Jervase J, Lawati AA. Solar radiation estimation using artificial neural networks. Appl. Energy 2002; 71:307-319.

[10] Mellit A, Benghanem M, Kalogirou SA. An adaptive wavelet-network model for forecasting daily total solar-radiation. Appl. Energy 2006; 83:705-722.

[11] Paoli C, Voyant C, Muselli M, Nivet M. Forecasting of preprocessed daily solar radiation time series using neural networks. Solar Energy 2010; 84:2146-2160.

[12] Chen JL, Liu HB, Wu W, Xie DT. Estimation of monthly solar radiation from measured temperatures using support vector machines-A case study. Renew Energy 2011; 36:413-420.

[13] Wu W, Liu HB. Assessment of monthly solar radiation estimates using support vector machines and air temperatures. Int. J. Climatol. 2012; 32:274-285.

[14] Cao J, Lin X. Study of hourly and daily solar irradiation forecast using diagonal recurrent wavelet neural networks. Energy Convers Manage 2008;49(6):1396-1406.

[15] Alamsyah TMI, Sopian K, Shahrir A. Predicting average energy conversion of photovoltaic system in Malaysia using a simplified method. Renew Energy 2003; 29:403-411.

[16] Ropp ME, Begovic M, Rohatgi A, Long R. Design considerations for large roofintegrated photovoltaic arrays. Prog Photovolt Res Appl 1997; 5:55-67.

[17] Dalton GJ, Lockington DA, Baldock TE. Feasibility analysis of renewable energy supply options for a grid-connected large hotel. Renew Energy 2009; 34:955-964.

[18] Bacher P, Madsen H, Nielsen HA. On-line short-term solar power forecasting. Solar Energy 2009; 83:1772-1783.

[19] Fernandez-Jimenez LA, Munoz-Jimenez A, Falces A, Mendoza-Villena M, Garcia-Garrido E, LaraSantillan PM, Zorzano-Alba E, Zorzano-Santamaria PJ. Short-term power forecasting system for photovoltaic plants. Renew Energy 2012; 44:311-317.

[20] Li YT, Shu LJ, Su Y. An ARMAX model for forecasting the power output of a grid connected photovoltaic system. Renew Energe 2014; 66:78-89.

[21] Wong S, Wan KK, Lam TN. Artificial neural networks for energy analysis of office buildings with daylighting. Appl Energy 2010; 87:551-517.

[22] Sulaiman SI, Abdul Rahman TK, Musirin I, Shaari S. Performance analysis of evolutionary ANN for 
output prediction of a grid-connected photovoltaic system. Int J Electr Comput Eng 2010; 5:244-249.

[23] Ding M, Wang L, Bi R. An ANN-based approach for forecasting the power output of photovoltaic system. Procedia Environ Sci 2011; 11:1308-1315.

[24] Chen CS, Duan SX, Cai T, Liu BY. Online 24-h solar power forecasting based on weather type classification using artificial neural network. Solar Energy 2011; 85:2856-2870.

[25] Mora-lopez L, Martinez-Marchena I, Piliougine M, Sidrach-deCardona M. Machine learning approach for next day energy production forecasting in grid connected photovoltaic plants. World Renew Energy Congress 2011; 2869-2874.

[26] Shin HJ. Partial functional linear regression. J Stat Plan Infer 2009; 139:3405-3418.

[27] Yao F, Müller HG, Wang JL. Functional data analysis for sparse longitudinal data. J. Am Stat Assoc 2005; 100:577-590.

[28] Wang GC, Feng XN, Chen M. Functional partial linear single-index model. Scandinavian Journal of Statistics 2016; 43: 261-274.

[29] Ash RB, Gardner MF. Topics in Stochastic Processes. New York: Academic Press 1975.

[30] Rice J., Silverman B. Estimating the mean and covariance structure nonparametrically when the data are curves. J. R Stat Soc B 1991; 53:233-243.

[31] Hadi AS, Ling RF. Some cautionary notes on the use of principal components regression. Am Stat 1998; 52:15-19.

[32] Bair E, Hastie T, Paul D, Tibshirani R. Prediction by supervised principal components. J. Am Stat Assoc 2006; 101:119-137.

[33] Zhu H, Vannucci M, Cox DD. Functional data classification in cervical pre-cancer diagnosis - a bayesian variable selection model. In Proc. Jt Statist. Meet 2007.

[34] Tibshirani R. Regression shrinkage and selection via a Lasso. J Royal Stat Soc B 1996; 58:267-288.

[35] Fu WJ. Penalized regressions: the bridge versus the lasso. J of comput graph stat 1998; 7:397-416.

[36] Fan J, Li R. Variable selection via nonconcave penalized likelihood and its oracle properties. J Am Stat Assoc 2001; 96:1348-1360.

[37] Efron B, Hastie T, Johnstone I, Tibshirani R. Least angle regression. Ann stat 2004; 32:407-499.

[38] Zou H, Hastie T, Tibshirani R. On the degrees of freedom of the lasso. Ann Stat 2007; 35:2173-2192.

[39] Yuan M, Lin Y. Model selection and estimation in regression with grouped variables. J R Stat Soc B 2006; 68:49-67.

[40] Zou H. The adaptive lasso and its oracle properties. J Am stat assoc 2006; 101:1418-1429. 